\title{
Observational constraints on massive star evolution
}

\author{
Philip Massey \\ Lowell Observatory, 1400 West Mars Hill Rd., Flagstaff, AZ86001, USA
}

\begin{abstract}
In this paper, I discuss the observational quantities that are useful for judging the successes and failures of current massive star evolutionary theory. The galaxies of the Local Group can serve as important laboratories for providing these diagnostics, as their metallicities vary by a factor of ten. We find that the evolutionary tracks do a good job of matching the distribution of stars in the H-R diagram during the main-sequence phase. However, none of the models produce RSGs that are as cool and as bright as what is observed. The relative number of WC and WN stars is a strong function of metallicity, and the Padova and Geneva 'normal mass-loss' models do a reasonably good job of matching the observations at low metallicities, but predict too few WCs at higher metallicity. The 'enhanced' mass-loss models of the Geneva group do not match the observations at all. New data is providing excellent statistics on the number of RSGs in these nearby galaxies, and the number ratio of RSGs to WRs is also an extremely sensitive function of metallicity. None of the models reproduce the trend of the RSG/WR ratio with metallicity.
\end{abstract}

\section{Introduction: using the Local Group as our laboratory}

The evolution of massive stars is hard to model due in large part to the complications of mass-loss. Stellar winds are constantly stripping the H-rich outer material from an O-type star's surface, and a very massive star might lose half of its mass during its main-sequence evolution. If a star undergoes a Luminous Blue Variable (LBV) phase, then mass-loss becomes episodic, making its inclusion in models even more difficult. The mass-loss rates during the red supergiant (RSG) phase are high, but are poorly understood. And, during the Wolf-Rayet (WR) phase mass-loss rates can be as much as an order of magnitude larger than during the main-sequence phase, due to lower surface gravity, higher abundances, and high effective temperatures. Efforts to model the evolution of massive stars are further hampered by uncertainties in mixing and convection, with most recent work directed towards the inclusion of rotation. Thus, feedback between stellar evolutionary theory and observational data is crucial, both for providing 'reality checks' on the models, and for directing observers in useful directions. One caveat in this is that we must make sure that the observational data are accurate, else we will lead our theoretician colleagues on a merry chase.

The galaxies of the Local Group provide ideal laboratories for exploring our ideas of massive star evolution: the stars are bright enough, and the galaxies are close enough, so that we can resolve the massive star population. The metallicity changes by a factor of ten among the galaxies that are currently forming stars, with the SMC and the Andromedea galaxy (M31) forming the two extremes. 
And, this is a wonderful time for such studies, with improved models becoming available, and with the advent of large Mosaic imagers on 4-m telescopes and multi-object spectrographs on 8-m telescopes.

There are two basic sorts of tests that we can perform. In the first of these we compare global properties, such as the number of RSGs to WRs, or WCs to WNs. Such tests rely upon two assumptions: $(i)$ that we have averaged over enough of a galaxy to be totally heterogeneous with respect to age; and (ii) that the initial mass function is the same in the regions we are comparing. The second sort of test is potentially more powerful, but more risky, and that is using coeval regions to determine the progenitor masses of evolved objects from the turn-off masses. This can give us more direct information, but we can be more easily fooled by non-coevality and the effects of small number statistics.

Some of the observational quantities that can be determined reliably at present are: $(i)$ the number of blue and red supergiants more luminous than $M_{\text {bol }}<-7.5$. Data on this exists for most of the Magellanic Clouds, and will shortly exist for the other galaxies in the Local Group currently forming stars (e.g., Andromeda, NGC 6822, IC 10, IC 1613, M33, WLM, Phoenix, and Pegasus). Note that identifying the number of blue supergiants is not the same as knowing the number of O-type stars; that number is unknown at present even for the LMC; (ii) the number of WR stars within these Local Group galaxies; and (iii) the turn-off mass in clusters containing WRs and LBVs in the Milky Way and Magellanic Clouds; further work is needed to quantify this in the more distant galaxies of the Local Group.

\section{Main-sequence evolution}

Stars spend $90 \%$ of their lives undergoing core- $\mathrm{H}$ burning on the main-sequence, and so if we get this phase of their lives right we have done very well. It is also obvious that there has to be good agreement between observation and theory here if there is to be any hope of matching the observations for evolved massive stars. The situation in fact appears to be very good, with several critical observational tests giving confidence that we know what is happening pretty well during the main-sequence. Let me mention a few such studies.

Meylan \& Maeder (1982) were the first to describe what has become known as the 'main-sequence widening' or as the 'blue Hertzsprung gap' problem. Using data in young Galactic and Magellanic Cloud clusters, they compared the number of stars in various parts of color-magnitude diagrams to that predicted by the evolutionary tracks. These regions were each presumed to be coeval. They found an overabundance of A-type supergiants, particularly at low metallicities. It is as if the true main-sequence extended all the way to A-type supergiants. Meylan \& Maeder (1982) suggested that including enhanced mixing in the models might extend the main-sequence to cooler effective temperatures, which was confirmed by Langer (1991). However, a recent study by the Padova group suggests that this problem is not a main-sequence problem at all. Rather, they have included improved (higher) mass-loss rates during the RSG phase, and find that there are very extended loops in the evolutionary tracks that allow a good match with the distribution of stars (Salasnich, Bressan \& Chiosi 1999).

Fitzpatrick \& Garmany (1990) used an updated catalog of LMC members to compare the distribution of stars in the H-R diagram to that predicted by 
evolutionary tracks. Here the situation was quite different than in the clusters studied by Meylan \& Maeder (1982), in that they were looking at a presumed mixed-age population. Their study suggested that blue loops were indeed needed to explain the abundance of stars beyond the main-sequence.

Massey et al. (1995) added several hundred more spectral types to the Fitzpatrick \& Garmany (1990) LMC sample, and extended the study to the SMC. They were perhaps the first to emphasize that a $15 \mathcal{M}_{\odot}$ A-type supergiant will be $\sim 3$ mag brighter visually than an $85 \mathcal{M}_{\odot}$ O-type star on the ZAMS, despite the former being 2 mag fainter bolometrically. Thus in any $V$-limited catalog the younger more massive stars will be under-represented relative to cooler stars. When the catalog numbers were corrected empirically using several deep 'incompleteness fields' there was excellent agreement between the number of stars and those predicted by the evolutionary models, with the possible exception of stars very near the ZAMS.

One nagging question about main-sequence evolution has been that of the 'mass discrepancy'. Herrero et al. (1992) first called attention to the fact that the masses derived from modern atmosphere models could be as much as a factor of two lower than that suggested by the masses inferred from evolutionary tracks. This problem has persisted to this day, and there is a special session organized around the problem at this symposium. Various authors have attempted to resolve this issue by using spectroscopic binaries to provide a 'true' estimate of the masses. Burkholder, Massey, \& Morrell (1997) found good agreement between the orbital masses and the evolutionary tracks up to $25 \mathcal{M}_{\odot}$. Although some higher mass systems showed lower masses than that expected from the tracks, these invariably were cases where the stars were filling their Roche lobes, and enhanced mass-loss could easily account for the results. Generally the problem could not be resolved unless some detached systems of higher mass could be found. Fortunately, Massey \& Hunter (1998) came across four suitable candidates in the R 136 cluster. Followup HST observations by Massey, Penny \& Vukovich (2002) obtained radial velocity curves and established that three were eclipsing, allowing precise orbital determinations and masses to be determined. One of these appears to be the highest mass binary ever found. A comparison with the evolutionary models suggests excellent agreement for these four systems.

At this time I can only conclude that the observations and theory match very well on the main-sequence!

\section{Evolved stars}

There are still fundamental basic questions that remain about massive star evolution past the main-sequence. We still do not know what mass ranges become Wolf-Rayet stars, LBVs, or RSGs, or how these mass ranges depend upon metallicity. Here is what I think we know and what we don't.

\subsection{Red supergiants}

For RSGs there are a couple of important points to ponder. First, if our knowledge of the effective temperature scale of $M$ supergiants is correct, then none of the evolutionary models extend to cool temperatures at sufficiently high mass. This is illustrated in Figure 1, and is probably related to the choice of the mixinglength parameter $\alpha$ ( $c f$. figure 15 of Maeder \& Meynet 1987). 

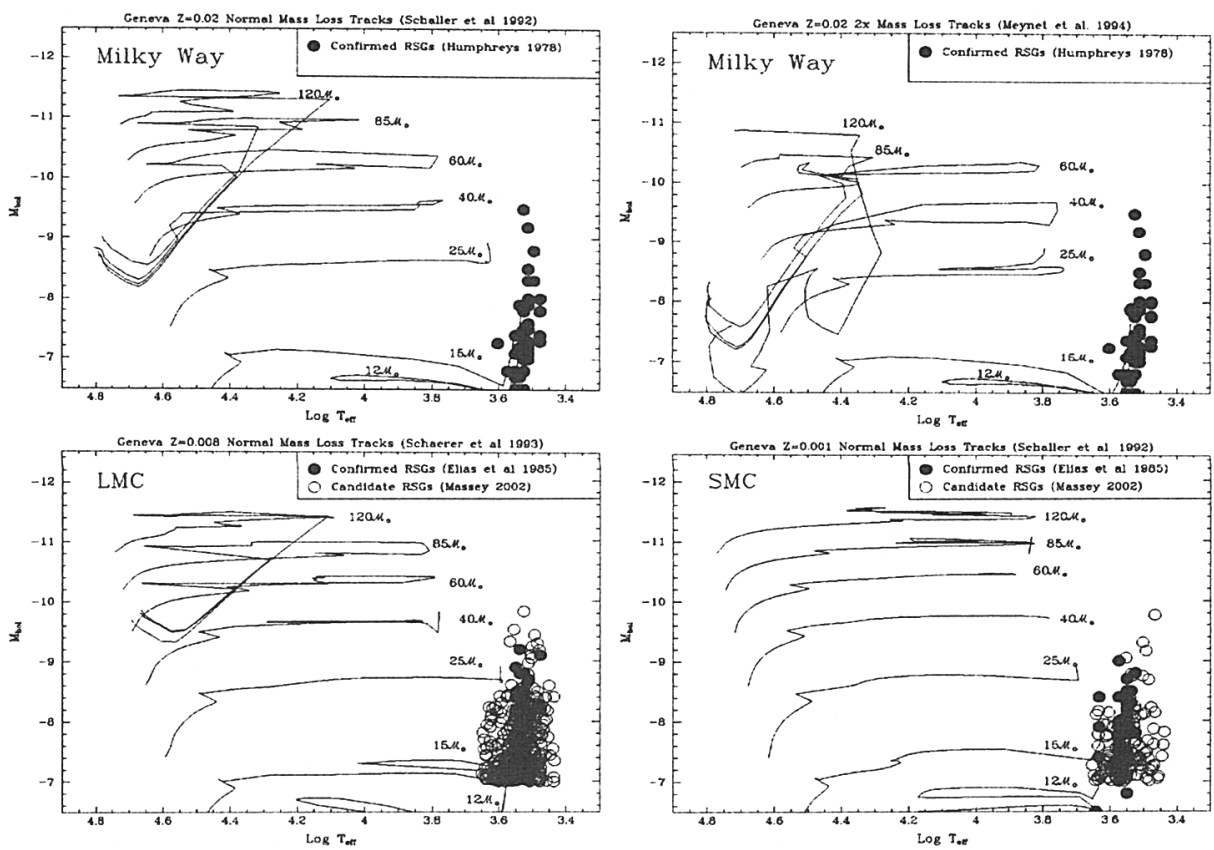

Figure 1. The location of RSGs is compared to the evolutionary tracks for the Milky Way, LMC, and SMC. In the upper two panels we use the $Z=0.02$ evolutionary tracks of the Geneva group; the tracks on the left have normal mass-loss rates (Schaller et al. 1992), and the ones on the right 'enhanced' rates (Meynet et al. 1994). Many of the RSGs (Humphreys 1978) are found at higher luminosities and cooler temperatures than the models allow. The bottom two panels show that the problem persists at low metallicity, where the RSGs come from Elias et al. (1985) and Massey (2002), and the tracks are those of $Z=0.008$ (Schaerer et al. 1993) and $Z=0.001$ (Schaller et al. 1992).

Secondly, we have begun to actually understand what the statistics are of RSGs in the Magellanic Clouds and other nearby galaxies. The key here is to properly remove the number of pesky foreground $M$ dwarfs (Massey 1998). If we do this we find something very interesting: the number of RSGs as function of luminosity changes drastically as a function of metallicity, with proportionately fewer of the highest luminosity RSGs found at higher metallicity. What was most surprising about this though, was that the higher luminosity RSGs did not simply vanish at higher $Z$; instead, there are just fewer of them. This suggests that what happens is that the proportional lifetimes of RSGs decrease at higher $Z$, in accord with what Maeder, Lequeux \& Azzopardi (1980) proposed.

Thirdly, there is the observation by Elias, Frogel \& Humphreys (1985) that the mean spectral type of RSGs changes with metallicity; i.e., that the average RSG is of somewhat earlier type in the SMC (M0) while in the Milky Way it is somewhat later (M2). This certainly suggests that if we are to compare the relative number of blue and red stars from galaxy to galaxy we have to be very careful what we count: for it to be meaningful we should include the $\mathrm{K}$ - and the M-type stars. Table 1 illustrates this from recent data on the Magellanic Clouds 
Table 1. Relative number of blue and red supergiants in the Magellanic Clouds

\begin{tabular}{lr}
\hline \hline Galaxy & B/R \\
\hline LMC (M) & 53 \\
SMC (M) & 165 \\
LMC (K+M) & 26 \\
SMC (K+M) & 20 \\
\hline \hline
\end{tabular}

(Massey 2002). If we count both the K- and M-type supergiants, there isn't much of a difference in the blue-to-red supergiant ratio for these two galaxies.

\subsection{Wolf-Rayet stars and LBVs}

Let us now turn to our attention to the Wolf-Rayet stars. The difficulties of being complete for the weaker-lined WNs have been most recently discussed by Massey \& Johnson (1998). Our knowledge of the WR content of the Magellanic Clouds is probably close to complete (Breysacher, Azzopardi \& Testor 1999; Massey \& Duffy 2001). Beyond the Magellanic Clouds, only a few small regions of NGC 6822, IC 1613, M33, and M31, and IC 10 have been surveyed in any detail (Massey \& Johnson 1998, and references therein).

We show in Figure 2 the relative number of WCs and WNs as a function of metallicity. There is a very smooth relationship, except for IC 10 and for the Milky Way data. The latter is probably incomplete. IC 10 is more of a puzzle. This galaxy is undergoing a starburst, and it is possible that something very peculiar related to star formation is going on. Alternatively it could just be that the data for IC10 are more incomplete than we've thought. Royer et al. (2001) found thirteen new WR candidates in this galaxy based upon imaging with interference filters. One of these turns out to be the already known WR star IC10-WR6 (Massey \& Armandroff 1995). Otherwise, none of the new candidates have been looked at spectroscopically. Massey \& Holmes (2002) have been analyzing a set of new very deep images and we detected seven of the remaining twelve Royer et al. (2001) candidates at high significance levels; the exceptions included all of their WC9 candidates. These don't show up as having $\lambda 4650$ emission in our survey. Massey \& Holmes also find a large number of other WR candidates. Spectroscopy is needed to confirm these, and determine their spectral subtype. So, I think it's premature to conclude that the IC 10 is telling us something fundamental.

We see in Figure 2 that the normal mass-loss models match the observations pretty well, although they predict too few WCs at high metallicities. The enhanced mass-loss rate models do not match the observations at all. In deriving the model predictions we have followed the precepts of Schaerer \& Vacca (1998), although we 'define' a WC star as a hot evolved star with a number ratio $\mathrm{C} / \mathrm{He}>0.1$ on the surface (Conti 1988; Crowther, Smith \& Willis 1995) rather than using the $\mathrm{C} / \mathrm{N}$ ratio.

Next, we can combine our results on the number of WRs and the number of RSGs; we show the results in Figure 3. We find a very clear trend with metallicity, with this ratio changing by two orders of magnitude over a change of $0.8 \mathrm{dex}$ in metallicity. None of the models reproduce this trend either. This 


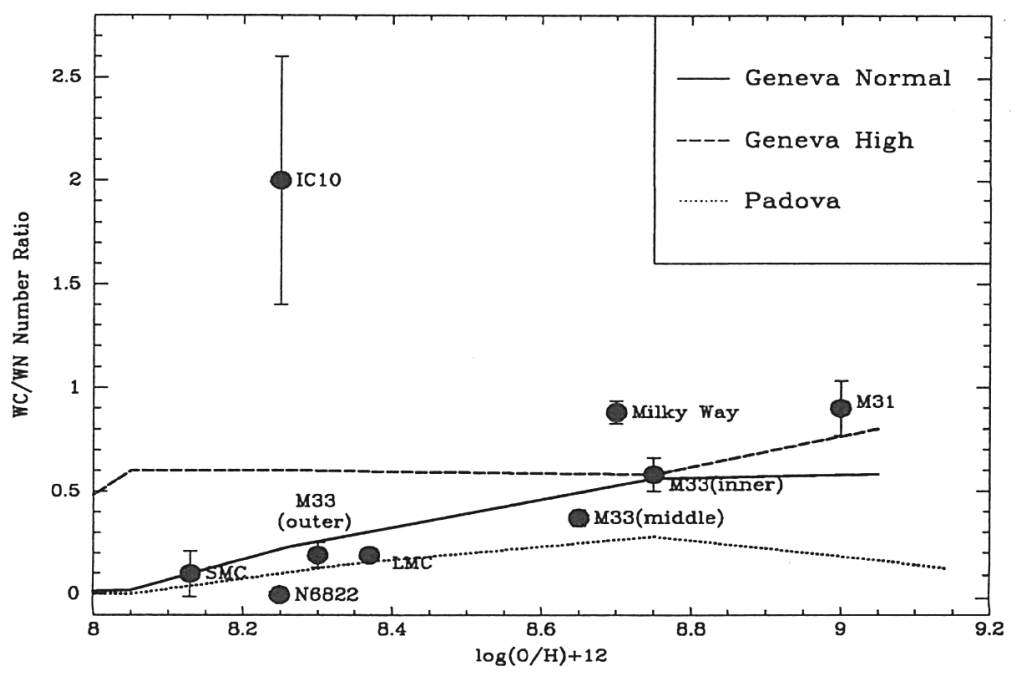

Figure 2. The relative number of WCs and WNs is shown as a function of metallicity. Recent work suggests that the IC 10 data may be incomplete. The predictions of the evolutionary models are also shown, with the 'Geneva Normal' referring to normal mass-loss rates, and the 'Geneva High' referring to the $2 \times$ enhanced mass-loss rates. The predictions from the Padova group are also shown. A Salpeter slope $\Gamma=-1.35$ and steady-state has been assumed.

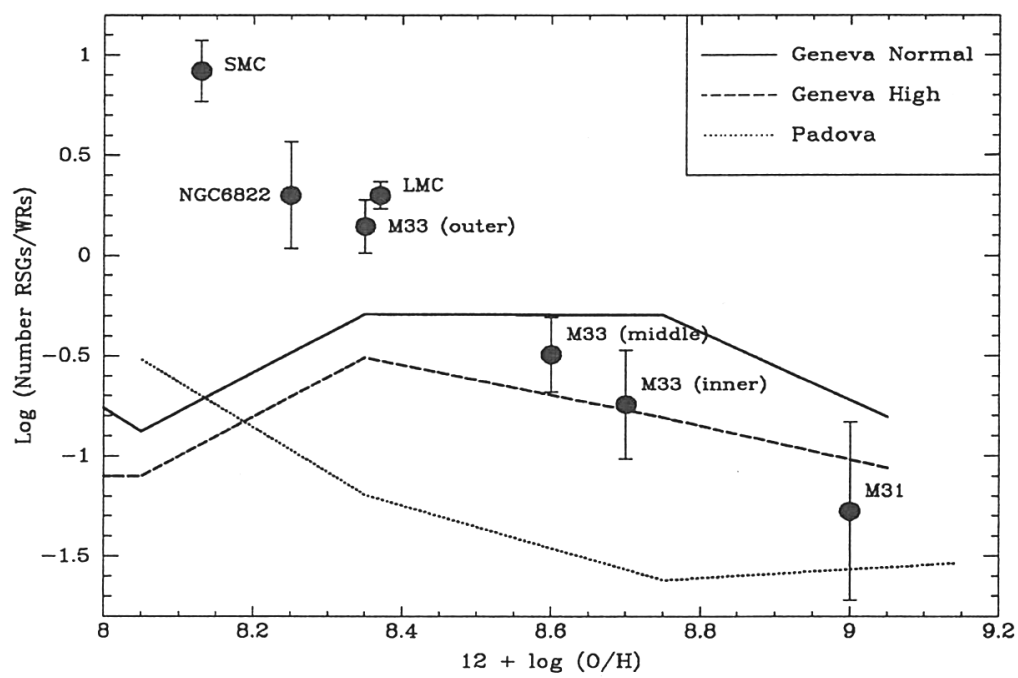

Figure 3. The relative number of RSGs and WRs as a function of metallicity.

is probably not surprising given the RSG problem pointed out in the previous section, and I believe that these new data offer a solid goal for new models to match.

Finally let me briefly summarize what I think we have learned recently from coeval regions in the MCs (Massey, Waterhouse \& DeGioia-Eastwood 2000; 
Massey, DeGioia-Eastwood \& Waterhouse 2001):

(i) in the SMC (low metallicity) Wolf-Rayet stars come only from the highest mass stars $\left(>85 \mathcal{M}_{\odot}\right)$, while in the higher metallicity Milky Way, we find early WNs in clusters with turn-off masses as low as 20-25 $\mathcal{M}_{\odot}$;

(ii) classical LBVs and WN7 stars are found only in clusters with very high turn-off masses, suggesting that they come from only the highest mass stars $\left(>120 \mathcal{M}_{\odot}\right)$; and

(iii) the Ofpe/WN9 stars are found in regions with relatively low turn-off masses $\left(<35 \mathcal{M}_{\odot}\right)$, suggesting that these are not related to the true LBVs.

Acknowledgments. This work has been supported most recently by the National Science Foundation, through grant AST-0093060. Useful comments came from Deidre Hunter, Kelsey Johnson, and particularly Daniel Schaerer.

\section{References}

Bohannan, B., Walborn, N.R. 1989, PASP 101, 520

Breysacher, J., Azzopardi, M., Testor, G. 1999, A\&AS 137, 117

Burkholder, V., Massey, P., Morrell, N. 1997, ApJ 490, 328

Conti, P.S. 1988, in: P.S. Conti \& A.B. Underhill (eds.), O Stars and Wolf-Rayet Stars, NASA SP-497, p. 81

Crowther, P.A., Smith, L.J., Willis, A.J. 1995, A\&A 304, 269

Elias, J.H., Frogel, J.A., Humphreys, R.M. 1985, ApJS 57, 91

Fitzpatrick, E.L., Garmany, C.D. 1990, ApJ 363, 119

Herrero, A., Kudritzki, R.P., Vílchez, J.M., et al. 1992, A\&A 261, 209

Humphreys, R.M. 1978, ApJS 38, 309

Langer, N. 1991, A\&A 252, 66

Maeder, A., Lequeux, J., Azzopardi, M. 1980, A\&A 90, 17

Maeder, A., Meynet, G. 1987, A\&A 182, 243

Massey, P., Armandroff, T.E. 1995, AJ 109, 2470

Massey, P., Bianchi, L., Hutchings, J.B., Stecher, T.P. 1996, ApJ 469, 629

Massey, P., Lang, C.C., DeGioia-Eastwood, K., Garmany, C.D. 1995, ApJ 438, 188

Massey, P. 1998, ApJ 501, 153

Massey, P., Johnson, O. 1998, ApJ 505, 793

Massey, P., Hunter, D.A. 1998, ApJ 493, 180

Massey, P., Waterhouse, E., DeGioia-Eastwood, K. 2000, AJ 119, 2214

Massey, P., DeGioia-Eastwood, K., Waterhouse, E. 2001, AJ 121, 1050

Massey, P., Duffy, A.S. 2001, ApJ 550, 713

Massey, P. 2002, ApJS 141, 81

Massey, P., Penny, L.R., Vukovich, J. 2002, ApJ 565, 982

Massey, P., Holmes, S. 2002, ApJ (Letters) 580, L35

Meylan, G., Maeder, A. 1982, A\&A 108, 148

Meynet, G., Maeder, A., Schaller, G., et al. 1994, A\&AS 103, 97

Royer, P., Smartt, S.J ., Manfroid, J., Vreux, J.-M. 2001, A\&A (Letters) 366, L1

Salasnich, B., Bressan, A., Chiosi, C. 1999, A\&A 342, 131

Schaerer, D., Meynet, G., Maeder, A., Schaller, G. 1993, A\&AS 98, 523

Schaerer, D., Vacca, W.D. 1998, ApJ 497, 618

Schaller, G., Schaerer, D., Meynet, G., Maeder, A. 1992, A\&AS 96, 269 


\section{Discussion}

LANGER: To what extent do you think are the lower limiting ZAMS single star masses for WR formation which you derive from your cluster observation affected by the fact that in close binaries WR stars can form from lower initial masses than in single stars?

MASSEY: It's an interesting question, isn't it, to what extent is WR formation affected by binary evolution. At the 1971 Wolf-Rayet meeting in Buenos Aires (IAU Symposium No. 49), before my time, there was unanimous agreement, I think, that all Wolf-Rayet stars formed by binary evolution. At the Qualicum Beach meeting (IAU Symposium No. 83, 1978) that was no longer the prevailing paradigm. Instead, though, we thought that maybe the newly found WRs in the SMC had formed by binary evolution, since many showed absorption lines in their spectra. Now we know that's not right either (see Foellmi et al. and Schnurr et al. in these Proceedings). Nichole King and I are also trying to answer this observationally. But the short answer to your question is that of course we worried about this; you can find more details in Massey et al. (2001, AJ 121, 1050). In a couple of cases the WR stars were binaries, with the companion's mass consistent with the masses inferred from other stars in the cluster. In most cases there is no evidence of radial velocity variation in the spectrum of the WR star and no spectroscopic sign of a companion. It would be a remarkable coincidence that the systematic trend with metallicity was due to binaries rather than mass-loss.

MEYNET: A small comment concerning the impact of rotation on the WR formation process. Rotation helps in forming WR stars by bringing at the stellar surface interior nuclear products. Due to this mixing mechanism, the star will have surface abundances equal to those of a WR star not as a result of heavy mass loss alone, but also due to the internal mixing induced by rotation. In addition, rotation models can also account for the existence of a WR star presenting both $\mathrm{H}$ - and He-burning products at their surface, namely ' $\mathrm{N}$ - and Ne-enhancements', a feature that non-rotating models cannot account for.

MASSEY: We all owe you and André Maeder a great deal of gratitude for these wonderful new models and I'm looking forward to using them.

CherepashChuK: The binary nature of $\eta$ Car seems to be proven by recent X-ray observations, according to Mike Corcoran (these Proceedings). What is your feeling about it?

MASSEY: I'm not sure that everyone in this room would necessarily agree that $\eta$ Car is a binary. (laughter.) But even if $\eta$ Car is a binary, so what? Does that necessarily mean that's why $\eta$ Car is an LBV? All I'll note is where $\eta$ Car sits in the H-R diagram of $\operatorname{Tr} 14 / 16$ is just what you'd expect for an evolved star in a very coeval region.

CROWTHER: $(i)$ I agree that we need to work hard on obtaining a genuine completeness with respect to WC/WN number ratios in external galaxies. For NGC 300 the WC/WN ratio has been revised from $\mathrm{WC} / \mathrm{WN}=2$ (high) to $\mathrm{WC} / \mathrm{WN}<1 / 3$ (oK) by Crowther et al. (these Proceedings). (ii) I disagree that all slash stars (Ofpe/WN9 or WN9-11) are 'false' LBVs with low initial masses: e.g., R 127 and AG Car are very luminous stars and most definitely 'genuine' LBVs. $\eta$ Car is the oddest LBV of all!

MASSEY: $(i)$ Well, while the discovery of Wolf-Rayet stars in NGC 300 by Breysacher et al. (1997, A\&A 326, 976) was very exciting, I don't think that anyone seriously thought the numbers were complete. For IC 10, I feel embarrassed, as I thought we had good evidence that we were complete - in particular, we had found WNs with as weak lines as any known in the SMC. But now Shay Holmes and I (Massey \& Holmes 2002) have found more WC and WN candidates. It's bursting even more than we thought. (ii) As 
for the slash stars, maybe the answer is that there are slash stars, and then there are slash stars. The two Ofpe/WN9 stars in our sample are found in coeval clusters with very modest turn-off masses and luminosities. $\eta$ Car may be an oddball, but S Doradus defines the class - and it (like $\eta$ Car) is found in a coeval region with a very high turn-off mass. My feeling is that this is telling us something.

ConTI: In response to one question back, there are two types of 'slash' stars! One group (e.g., in NGC 3603) is quite luminous. Phil's two Ofpe/WN objects are quite faint.

MASSEY: The two Ofpe/WN9 objects in our sample were Brey $18\left(\mathrm{M}_{V}=-6.6\right)$ and BE $381\left(M_{V}=-5.8\right)$. It's hard to know how to compare these to R127, since the latter is variable and its spectrum (pre-outburst) not all that similar (Bohannan \& Walborn 1989).

WALBORN: You're making an admirable dent in the nearby Universe with these massive surveys, which are also discovering numerous interesting individual objects. With regard to the relationship between Ofpe/WN9 stars and LBVs, it is true that some (subsets) of the class have relatively low masses (e.g., R 84 in a B-type cluster, as shown by H. Schild some time ago). However, R 127 was one of the origional Ofpe/WN9 stars; it is currently in an LBV outburst with an A-type spectrum identical to that of S Doradus, and it is the visually brightest star in the LMC. It is in a small cluster currently under investigation by Heydari-Malayeri et al., which contains two Of supergiants and must have a high turn-off mass.

MASSEY: Thanks for the kind words. When we did our study of coeval regions in the Magellanic Clouds (Massey et al. 2000) we used fairly stringent criteria for what regions to include. R 127 was not in a catalogued OB association, and we didn't include it. Of the two slash stars that were in our sample, one is BE 381, which also is one of the origional Ofpe/WN9 stars (Bohannan \& Walborn 1989) - if any star is the prototype for this class, this is it. By contrast, the spectrum of R 127 you show in Bohannan $\&$ Walborn is rather unlike that of the other slash stars, with no sign of He I P-Cygni profiles. In Massey et al. (1996) we divide up the slash stars into 'strong-lined' and 'weak-lined' - BE 381 clearly falls in to the former category, while I would have put $\mathrm{R} 127$ into the second catagory, in its pre-outburst phase.

SCHAERER: For 'numerical' reasons, discussed in Schaerer \& Vacca (1998), I think that your conclusion on the comparison of WC/WN number ratios with high mass loss models is not rubust. On the other hand, there is now a large number of WR galaxies (over a large metallicity range) which show WC/WN ratios requiring enough WC stars as in non-rotating high-mass loss tracks or, hopefully, also explained by models with rotation.

MASSEY: In our correspondence before the meeting, you very kindly reminded me of the need to interpolate the surface composition along an isochrone to determine when you have a WR and when you don't. I therefore followed your methodology, but with improved time sampling and using the recent abundance studies by Paul Crowther for deciding where to draw the line between a WC and an WN. In making the comparisons, I assumed steady-state star formation (appropriate to a heterogeneous mix of ages), and adopted a Salpeter IMF slope. In retrospect, maybe the 'enhanced' mass-loss models were developed in order to match a 'bad' observational quantity. Certainly, the normal (un-enhanced) mass-loss stellar evolutionary models do better at matching a number of well-determined observational quantities. It will be great when the rotating models are available for similar comparisons to be made. 